\title{
Numerical modeling of the electron temperature crashes observed in Wendelstein
}

\section{7-X stellarator experiments}

Q. Yu ${ }^{1,}$, E. Strumberger ${ }^{1}$, V. Igochine ${ }^{1}$, K. Lackner ${ }^{1}$, H.P. Laqua ${ }^{2}$, M. Zanini ${ }^{2}$, H. Braune ${ }^{2}$, M. Hirsch $^{2}$, U. Höfel ${ }^{2}$, S. Marsen ${ }^{2}$, T. Stange ${ }^{2}$, R.C. Wolf ${ }^{2}$, S. Günter ${ }^{1,2}$, and the Wendelstein 7-X Team**

${ }^{1}$ Max-Planck-Institut für Plasmaphysik, 85748 Garching, Germany ${ }^{2}$ Max-Planck-Institut für Plasmaphysik, 17491 Greifswald, Germany

Numerical calculations based on nonlinear two-fluid equations have been carried out to understand the sawtooth-like crashes of electron temperature observed in Wendelstein 7-X stellarator experiments with electron cyclotron current drive (ECCD). The application of ECCD leads to nonmonotonic radial profiles of the safety factor $\mathrm{q}$ with two $\mathrm{q}=1$ surfaces in the plasma core region. Using input parameters similar to the experiment, numerical calculations show two types of crashes of the central electron temperature depending on the growth of the internal kink mode coupled to double tearing modes. When the distance between two equilibrium $q=1$ surfaces is sufficiently large, the internal kink mode leads to a fast radial displacement of the hot core from the center to the region of the $\mathrm{q}=1$ surfaces. In agreement with experimental observation, this causes a full crash of central electron temperature in about 20-30 microseconds. When the distance between the two $q=1$ surfaces is not large enough, partial crashes of the central electron temperature are found. During these crashes the hot core only temporally moves outwards. Then it moves back, because the double tearing mode perturbation leads to an increase of the minimum q value about one.

Key words: internal kink mode, double tearing modes, sawtooth crash, stellarator, magnetic island, two-fluid equations.

*E-Mail: qingquan.yu @ipp.mpg.de

***For Wendelstein 7-X Team, see author list: T. Klinger et al 2019 Nucl. Fusion 59112004 


\section{Introduction}

In Wendelstein 7-X (W7-X) stellarator experiments, electron cyclotron current drive (ECCD) is a possible way to compensate the bootstrap current for controlling the island divertor configuration and the strike line location on divertor plates [1]. As the bootstrap current is larger for higher plasma pressure, ECCD could be crucial for achieving high plasma performance together with optimal divertor configuration.

Without applying ECCD, the magnetic shear in W7-X experiments is very low, and the radial profile of the rotational transform $\mathrm{l}$ is monotonic. In the case of slightly off-axis ECCD in co-direction, the 1 -profile can become non-monotonic with two $\mathrm{l}=1 / \mathrm{q}=1$ surfaces located in the plasma core region, because it increases iota and decreases the safety factor, q, respectively. In these co-direction ECCD experiments, strong oscillations of the electron temperature, $T_{e}$, have been observed, with a phase of slowly rising $\mathrm{T}_{\mathrm{e}}$ followed by a sudden crash in tens of microseconds ( $\mu \mathrm{s}$ ) [2,3], similar to the sawteeth observed in tokamak experiments [e.g. 4]. The inversion radius of the $\mathrm{T}_{\mathrm{e}}$ crashes approximately agrees with the radial location of $q=1$ surfaces $[2,3]$. An example of the experimental results for the crash of the central electron temperature is shown in figure 1, occurring in a time interval $\sim 25 \mu$ s between the two vertical dashed lines.

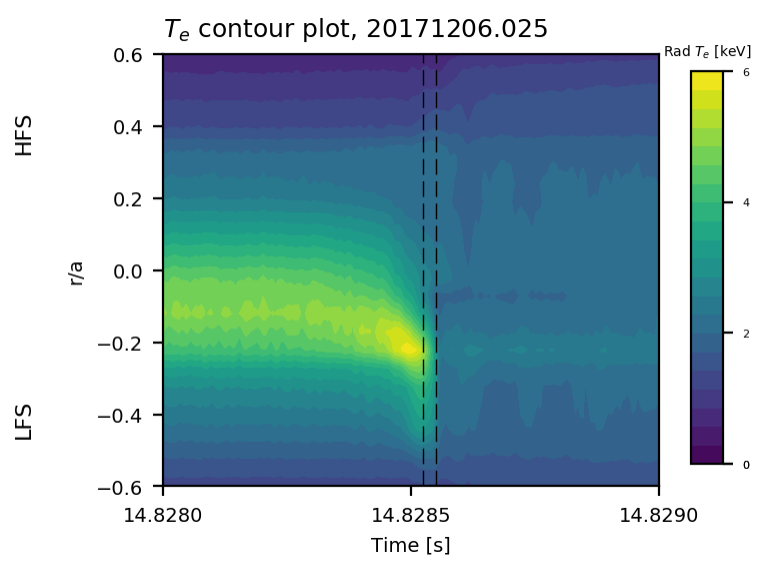

FIG. 1. An example of the radiation electron temperature observed by electron cyclotron emission (ECE) diagnostic in W7-X experiment with co-direction ECCD. Here r/a is the normalized effective minor radius. The fast crash phase is marked by the two vertical dashed lines, with a time interval $25 \mu$ s.

Linear, resistive stability studies were performed for W7-X equilibria with current drive, using the 3D CASTOR3D code [5]. Double tearing modes and internal resistive kink modes were found for iota-profiles modified by ECCD. Calculations using the CASTOR3D code have also been carried out for equilibria with the same 1-profiles, but with zero plasma pressure and a simplified geometry, namely a periodical cylinder. It turns out that the geometry does not influence the general stability 
behavior much, so that the growth rates obtained for 3D and cylindrical symmetry are similar.

For a better understanding of the observed crashes, it is desirable to simulate the nonlinear evolution of the instabilities. The initial value code TM1 is a possible choice for this purpose, since it can calculate MHD instabilities using realistic experimental parameters based on nonlinear two-fluid equations, including the effects of electron inertia, diamagnetic drifts and the associated ion polarization current $[6,7]$. However, a large aspect ratio and circular equilibrium magnetic surfaces are assumed in TM1. While the aspect ratio in $\mathrm{W} 7-\mathrm{X}$ is sufficiently large [2,3], the magnetic field geometry is much more complex. In particular the shaping of the magnetic surfaces and mode coupling due to the stellarator symmetry cannot be taken into account. Nevertheless, due to the agreement in the linear studies between the realistic and the simplified geometry, and the fact that low $\mathrm{m} / \mathrm{n}$ modes play the major role $(\mathrm{m} / \mathrm{n}$ is the poloidal/toroidal mode number), TM1 might still capture the major features of the experimental observations.

Usually, the q-profile is directly related to the profile of the plasma current density in any axissymmetric geometry. To deal with a stellarator plasma, the TM1 code has been modified such that except for the current driven by ECCD, the poloidal equilibrium magnetic field is generated by a coil current rather than by the plasma current. With this new version of the TM1 code, nonlinear simulations have been carried out. The major numerical results are reported in this paper. Sawtoothlike crashes of the electron temperature are found in the numerical calculations.

In the next section our theoretical model is described. The numerical results are presented in Section 3, followed by discussion and summary in the last section.

\section{Numerical model}

The toroidal mode coupling is neglected, which is appropriate for the modes with low mode numbers due to the small variation of the experimental $q$ value along the minor radius. The magnetic field is defined as

$$
\mathbf{B}=\mathrm{B}_{\mathrm{t}} \mathbf{e}_{\mathrm{t}}-\left(\mathrm{k}_{\mathrm{t}} / \mathrm{k}_{\theta}\right) \mathrm{B}_{\mathrm{t}} \mathbf{e}_{\theta}+\nabla \psi \times \mathbf{e}_{\mathrm{t}},
$$

where $\mathrm{B}_{\mathrm{t}}$ is the toroidal magnetic field, $\psi$ is the helical flux function of the minor radius $\mathrm{r}$ and the helical angle, $\mathrm{k}_{\theta}=\mathrm{m} / \mathrm{r}$ and $\mathrm{k}_{\mathrm{t}}=\mathrm{n} / \mathrm{R}_{0}$ are the wave vector in $\mathbf{e}_{\theta}$ (poloidal) and $\mathbf{e}_{\mathrm{t}}$ (toroidal) direction, and $\mathrm{R}_{0}$ is the major radius of the magnetic axis. While the equilibrium helical flux in a tokamak is produced by the plasma current, in a current-free stellarator it is produced by external coil currents except for that from ECCD. Therefore, the helical flux function has three parts,

$$
\psi=\psi_{\text {coil }}+\psi_{\mathrm{ECCD}}+\psi_{\text {pert }}
$$

corresponding to the additional coil currents, ECCD and the perturbation caused by instabilities. In our 
numerical code the spatial location of the coils is inside the plasma region, but the coils are assumed to occupy zero space and to have no interaction with the plasma except for generating the required equilibrium poloidal field.

The total parallel current density is described by

$$
\mathrm{j}_{\text {total }}=\mathrm{j}_{\text {coil }}+\mathrm{j} \text {, }
$$

where $j_{\text {coil }}$ is the coil current density being constant in time, and

$$
\mathrm{j}=\mathrm{j}_{\mathrm{ECCD}}+\mathrm{j}_{\text {pert }} \text {, }
$$

including the plasma current density due to ECCD and instabilities.

In order to study the nonlinear evolution of the instabilities, the two-fluid equations used here include the continuity equation, the generalized Ohm's law, the equation of motion in the perpendicular (after taking $\mathbf{e}_{t} \cdot \nabla \times$ ) and the parallel (to magnetic field) direction, and the electron heat transport equation. Normalizing the length to the minor radius $a$, the time $t$ to the resistive time $\tau_{R}=a^{2} \mu_{0} / \eta$, the helical flux $\psi$ to $\mathrm{aB}_{\mathrm{t}}$, the ion velocity $\mathbf{v}$ to $\mathrm{a} / \tau_{\mathrm{R}}$, and the electron density $\mathrm{n}_{\mathrm{e}}$ and $\mathrm{T}_{\mathrm{e}}$ to their values at the magnetic axis, these equations become [6,7]

$$
\begin{aligned}
& \frac{d n_{e}}{d t}=d_{1} \nabla_{||} j-\nabla_{\|}\left(n_{e} v_{\|}\right)+\nabla_{\perp}\left(D_{\perp} \nabla_{\perp} n_{e}\right)+S_{n}, \\
& \frac{d \psi}{d t}=-\eta j_{p e r t}-\frac{\eta}{v_{e i}} \frac{d j}{d t}++\Omega\left(\frac{T_{e}}{n_{e}} \nabla_{\|} n_{e}+\nabla_{\|} T_{e}\right) \\
& \frac{d U}{d t}=-S^{2} \nabla_{||} j+\mu \nabla_{\perp}^{2} U \\
& \frac{d v_{\|}}{d t}=-C_{S}^{2} \nabla_{\|} P / n_{e}+\mu \nabla_{\perp}^{2} v_{\|}, \\
& \frac{3}{2} n_{e} \frac{d T_{e}}{d t}=d_{1} T_{e} \nabla_{||} j-T_{e} n_{e} \nabla_{\|} v_{\|}+n_{e} \nabla \cdot\left(\chi_{\|} \nabla_{\|} T_{e}\right)+n_{e} \nabla \cdot\left(\chi_{\perp} \nabla_{\perp} T_{e}\right)+S_{p}
\end{aligned}
$$

where

$$
\mathrm{d} / \mathrm{dt}=\partial / \partial \mathrm{t}+\mathbf{v}_{\perp} \cdot \nabla_{\perp}
$$

and

$$
\mathrm{U}=-\nabla_{\perp}^{2} \phi
$$

is the plasma vorticity, $\phi$ is the stream function, $\mu$ the plasma viscosity, $\chi$ the heat conductivity, and $\mathrm{D}$ the particle diffusivity. $\mathrm{P}=\mathrm{P}_{\mathrm{e}}=\mathrm{n}_{\mathrm{e}} \mathrm{T}_{\mathrm{e}}$, and the subscripts $\|$ and $\perp$ denote the parallel and the perpendicular components, respectively. $S_{n}$ and $S_{p}$ are the particle and heat sources. The parameters in equations (5)(9) are given by $d_{1}=\omega_{c e} / v_{e i}, \Omega=\beta_{e} d_{1}, C_{s}=\left[T_{e} / m_{i}\right]^{1 / 2} /\left(a / \tau_{R}\right)$, and $S=\tau_{R} / \tau_{A}$, where $\beta_{e}=4 \pi n_{e} T_{e} / B_{0 t}{ }^{2}, \omega_{c e}$ is the electron cyclotron frequency, $v_{\mathrm{ei}}$ is the electron-ion collisional frequency, and $\tau_{\mathrm{A}}=\mathrm{a} / \mathrm{V}_{\mathrm{A}}$ is the toroidal Alfven time. $C_{s}$ and $\eta$ are the normalized ion sound velocity and resistivity. The cold ion assumption is made. The second term on the right hand side of equation (6) takes into account the electron inertia. Equations (5)-(11) are solved simultaneously using the modified version of TM1. The previous 
version of the code had been successfully used for modelling fast sawtooth crashes for realistic tokamak parameters $[6,7]$.

Without ECCD, the 1 value of the considered W7-X discharge monotonically decreases from the edge towards the center. This corresponds to an increase of the q value from 1.03 to 1.15 , as shown by the dotted curve in figure 2. In order to simulate the effect of ECCD, an analytical function is added to the monotonic iota-profile $1_{0}$,

$$
\imath(\Phi)=l_{0}(\Phi)+\alpha_{0} \exp \left\{-\left[(\Phi-0.1) / \alpha_{1}\right]^{2}\right\},
$$

where $\Phi$ is the normalized toroidal magnetic flux. With increasing values of $\alpha_{0}$ and $\alpha_{1}$, the q-profiles become more non-monotonic with a larger radial distance between two $\mathrm{q}=1$ surfaces in the core region. Four examples are shown by the solid black, red, blue and green curves for different $\alpha_{0}$ and $\alpha_{1}$ values in figure 2.

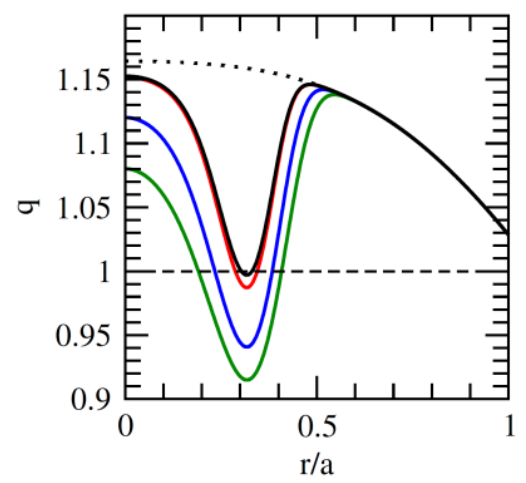

FIG. 2. Radial profiles of the equilibrium safety factor $q$. The dotted curve is the original q-profile without ECCD. The solid curves are for $\alpha_{0}=0.14$ and $\alpha_{1}=0.06$ (black), $\alpha_{0}=0.15$ and $\alpha_{1}=0.06$ (red), $\alpha_{0}=0.2$ and $\alpha_{1}=0.075$ (blue), and $\alpha_{0}=0.23$ and $\alpha_{1}=0.09$ (green). The horizontal dashed line shows the $q=1$ value.

\section{Numerical results}

The input parameters for our calculations, including the equilibrium electron temperature and density profiles, are taken from experimental data with the toroidal magnetic field $\mathrm{B}_{0 t}=2.45 \mathrm{~T}$, $\mathrm{T}_{\mathrm{e}}=3.18 \mathrm{keV}$, and $\mathrm{n}_{\mathrm{e}}=2.5 \times 10^{19} \mathrm{~m}^{-3}$ at the magnetic axis. The plasma minor radius is $\mathrm{a}=0.502 \mathrm{~m}$, and the aspect ratio $\mathrm{R}_{0} / \mathrm{a}=11$. These parameters lead to $\mathrm{S}=7.1 \times 10^{8}, \mathrm{C}_{\mathrm{s}}=5.97 \times 10^{7}\left(\mathrm{a} / \tau_{\mathrm{R}}\right), \Omega=2.4 \times 10^{5}$, and $\mathrm{d}_{1}=9.2 \times 10^{7}$. The electron density profile is flattening in the core region. The perpendicular plasma momentum, particle and heat transport are assumed to be at an anomalous transport level of $\mu=\mathrm{D}_{\perp}=$ $\chi_{\perp}=0.2 \mathrm{~m}^{2} / \mathrm{s}=37.6\left(\mathrm{a}^{2} / \tau_{\mathrm{R}}\right)$, and $\chi_{\|} / \chi_{\perp}=10^{9}$ is taken. A larger plasma viscosity for the $\mathrm{m} / \mathrm{n}=0 / 0$ component, $\mu_{0 / 0}=37.6 \times 10^{2}\left(\mathrm{a}^{2} / \tau_{\mathrm{R}}\right)$, is used in equation (7) to take into account the neoclassical damping of plasma rotation generated by instabilities [6]. The bootstrap current is negligible in the core region of these discharges and has not been included in calculations. 


\subsection{Linear results}

Linear stability studies were performed for W7-X equilibria with current drive using the CASTOR3D code [5]. While these calculations took into account the 3D geometry of W7-X, benchmark calculations have been made with CASOTR3D and TM1 codes for simplified cylinder geometry. For this comparison the single fluid version of the TM1 code is used, the rotational transform is solely provided by the plasma current, and the input parameters are taken to be $S=4.75 \times 10^{7}$, $\langle\beta\rangle=C_{s}=\Omega=d_{1}=0$, and $\mu=D_{\perp}=\chi_{\perp}=0$, where $\langle\beta\rangle$ is the volume averaged beta value. Figure 3 shows the linear growth rates obtained for the equilibrium with the q-profile depicted by the red curve in figure 2. The double tearing modes (DTMs) are found to be linearly unstable for toroidal mode numbers up to $n=16(m=n)$. The results of the two codes (black and red curves) agree very well. The green curve corresponds to the case with $\langle\beta\rangle=0.26 \%$, while the blue points are obtained for the realistic stellarator geometry with $\langle\beta\rangle=0$ by CASTOR3D. For the latter case, the rotational transform is provided by the external coil and ECCD currents. No big difference in simulation results is found from figure 3 between the simplified cylindrical and realistic stellarator geometry. This motivates us to further perform the two-fluid and non-linear simulations in the simplified geometry with TM1.

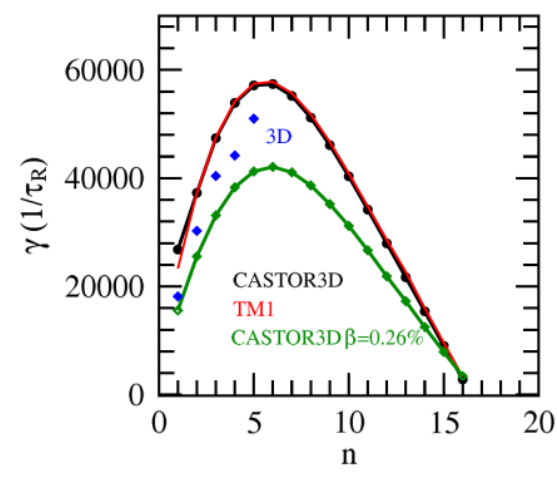

FIG. 3. Liner growth rates versus toroidal mode number $n$ obtained from CASTOR $3 D$ (black curve for $<\beta>=0$ and green curve for $\langle\beta\rangle=0.26 \%$ ) and TM1 (red for $\langle\beta\rangle=0$ ) for the single fluid case in the cylinder geometry. The blue points are obtained from CASTOR3D with experimental geometry for $\langle\beta\rangle=0$. Other input parameters are $S=4.75 \times 10^{7}$ and $C_{s}=\Omega=d_{1}=\mu=D_{\perp}=\chi_{\perp}=0$.

For the following results the "internal coil" current described in Section 2 is taken into account in the simplified cylindrical geometry, so that the equilibrium plasma current is only from the ECCD. Using the above mentioned experimental data as input and the q-profile shown by the red curve in figure 2, the linear mode growth rate obtained from TM1 for the single (red curve) and two fluid (black) cases is shown as a function of the toroidal mode number in figure 4 . The results obtained from the single fluid equations have about the same spectrum of unstable modes as that shown in figure 3 . The 
results obtained from two-fluid equations are quite different. The spectrum of unstable modes is shifted towards higher mode numbers, with the maximum growth rate around $n=10$. For low-m $(=n)$ modes, the difference between the single and two fluid cases is smaller.

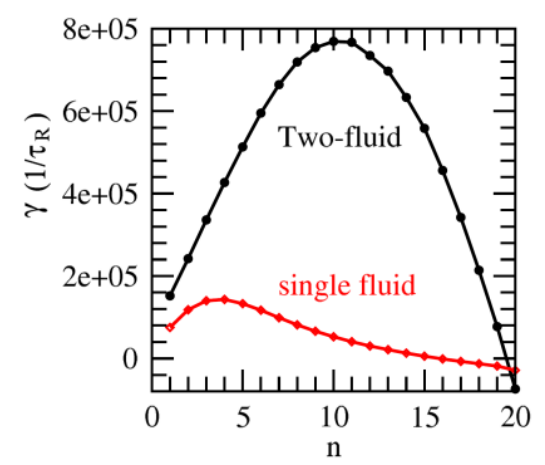

FIG. 4. Linear mode growth rate versus toroidal mode number $n(=m)$ obtained from TM1 for the single (red curve) and two-fluid case (black) with the q-profile shown by the red curve in figure 2.

\subsection{Nonlinear results with a small distance between the resonant surfaces}

In the nonlinear calculations, 30 Fourier components, including $m / n=0 / 0,1 / 1,2 / 2,3 / 3 /, \ldots$, as well as the interactions among them are included. The numerical convergence was checked with 60 Fourier components and the radial grid size as small as $3 \times 10^{-4} \mathrm{a}$.

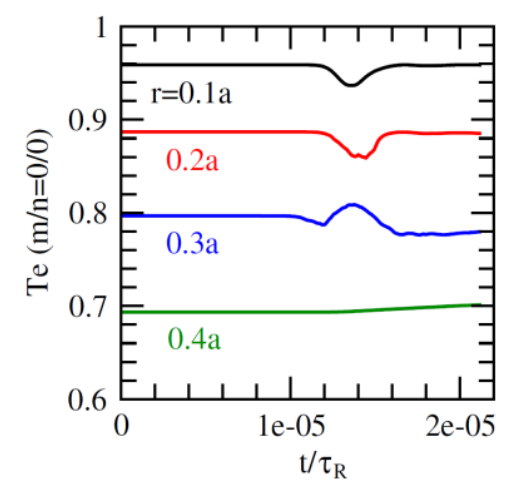

FIG. 5. The time evolution of the $m / n=0 / 0$ component of the electron temperature, $T_{e}(\mathrm{~m} / \mathrm{n}=0 / 0)$, at the radial location r/a=0.1, 0.2, 0.3 and 0.4, with the equilibrium q-profile shown by the red curve in figure 2.

Corresponding to the original equilibrium q-profile shown by the red curve in figure 2 , the time evolution of the $\mathrm{m} / \mathrm{n}=0 / 0$ component electron temperature, $\mathrm{T}_{\mathrm{e}}(\mathrm{m} / \mathrm{n}=0 / 0)$, at the radial location $\mathrm{r} / \mathrm{a}=0.1$, $0.2,0.3$, and 0.4 is shown in figure 5 . As the initial perturbations for all modes are set to be very small in the calculation, the unstable modes need time to grow up to a sufficiently large amplitude to cause a visible change in the electron temperature (until $\mathrm{t}=10^{-5} \tau_{\mathrm{R}}, \tau_{\mathrm{R}}=47 \mathrm{~s}$ ). These changes first appear around the $\mathrm{q}=1$ surfaces (blue curve). The two $\mathrm{q}=1$ surfaces of the equilibrium q-profile are at $\mathrm{r} / \mathrm{a}=0.287$ and 0.344. About $90 \mu$ s later (around $t=1.2 \times 10^{-5} \tau_{R}$ ), the electron temperature begins to decrease in the 
central region $(\mathrm{r} / \mathrm{a}=0.1$ and 0.2$)$, but increases at $\mathrm{r} / \mathrm{a}=0.3$ due to the heat flux resulting from an internal kink mode (IKM). Shortly afterwards, however, the central electron temperature recovers, while the electron temperature at $\mathrm{r} / \mathrm{a}=0.3$ remains to be lower than its original equilibrium value.

Corresponding to the results shown in figure 5, radial profiles of the radial ion velocity of the $\mathrm{m} / \mathrm{n}=2 / 2,3 / 3,4 / 4$, and $5 / 5$ modes are shown in figure 6 at a time well before the change of the electron temperature profile $\left(\mathrm{t}=5.91 \times 10^{-6} \tau_{\mathrm{R}}\right)$. The solid (dotted) curve show the real (imaginary) part. Due to the larger linear growth rate of higher-m modes as shown by the black curve in figure 4, their perturbation amplitude is larger than that of lower-m ones at these early times. The $\mathrm{m} / \mathrm{n}=1 / 1$ component is not shown in the figure, since its amplitude is even smaller than that of the $2 / 2$ component.

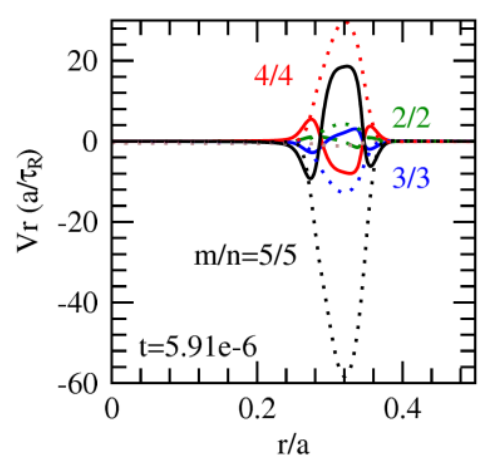

FIG. 6. Corresponding to the results shown in figure 5, radial profiles of the radial ion velocity of the $m / n=2 / 2$, $3 / 3,4 / 4$ and $5 / 5$ components at $t=5.91 \times 10^{-6} \tau_{R}$. The solid (dotted) curve shows the real (imaginary) part. Due to the larger linear growth rate of higher $m$ modes, their amplitude is larger. The $m / n=1 / 1$ mode is not shown, since its amplitude is even smaller than that of the 2/2 mode.

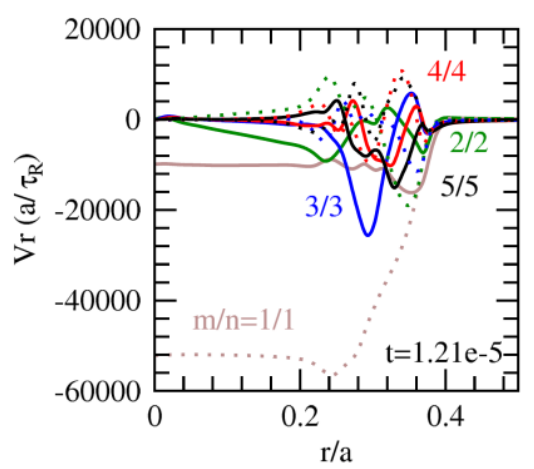

FIG. 7. Same as figure 6 but at a later time $\left(t=1.21 \times 10^{-5} \tau_{R}\right)$ right before the decrease of the central electron temperature. The $m=1$ component becomes dominant in the central plasma region.

At a later time $\left(\mathrm{t}=1.21 \times 10^{-5} \tau_{\mathrm{R}}\right)$ right before the drop of the central electron temperature, the radial profiles of the radial ion velocity of the $m / n=1 / 1,2 / 2,3 / 3,4 / 4$, and $5 / 5$ components are shown in figure 7. At this time the $\mathrm{m}=1$ component becomes the dominant mode in the central plasma region. The $2 / 2$ component perturbation also extends to the plasma center, while the higher-m components are still localized in the region between the two $q=1$ surfaces. During the non-linear evolution, the mode 8 
spectrum thus changes significantly compared to the linear phase, with a strongly driven $1 / 1$ IKM in the non-linear phase.

Corresponding to the simulations shown in figure 5, contours of constant helical flux (left) and electron temperature (right) are shown in figure 8 at $\mathrm{t}=1.05 \times 10^{-5}, 1.21 \times 10^{-5}, 1.37 \times 10^{-5}, 1.53 \times 10^{-5}$, and $1.69 \times 10^{-5} \tau_{\mathrm{R}}$ (from top to bottom). At $\mathrm{t}=1.05 \times 10^{-5} \tau_{\mathrm{R}}$ magnetic islands (DTMs) develop around the two $\mathrm{q}=1$ surfaces at $\mathrm{r} / \mathrm{a}=0.287$ and 0.344 . The high-m modes affect the magnetic island structure, leading to a strong localization in poloidal direction. At a later time $\left(\mathrm{t}=1.21 \times 10^{-5} \tau_{\mathrm{R}}\right)$, in addition to the DTMs, an IKM causes an outwards shift of the original plasma core, leading to a drop of the central electron temperature, as shown in figure 5. During the temperature crash phase, the IKM is dominated by the $\mathrm{m} / \mathrm{n}=1 / 1$ component, which has a larger amplitude than the other modes in the core region. The DTMs are localized around $\mathrm{q}=1$ surfaces and contain many components, such as $\mathrm{m} / \mathrm{n}=1 / 1,2 / 2,3 / 3, \ldots$, similar to the case shown in figure 7 . The core stops its outwards motion around $t=1.37 \times 10^{-5} \tau_{R}$ and then moves inwards, as can be seen when comparing the two contours at $t=1.37 \times 10^{-5}$ and $1.53 \times 10^{-5} \tau_{R}$. On a longer time scale the mode saturates as DTMs as shown by the contour at $t=1.69 \times 10^{-5} \tau_{R}$, and the $1 / 1$ component decays to a smaller amplitude becoming comparable to that of other components.
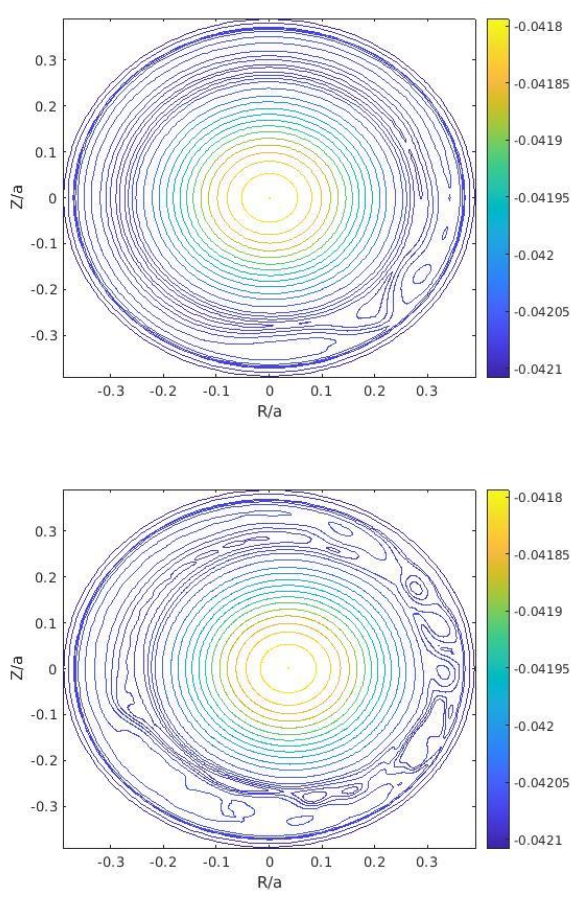

a) $t=1.05 \times 10^{-5} \tau_{R}$

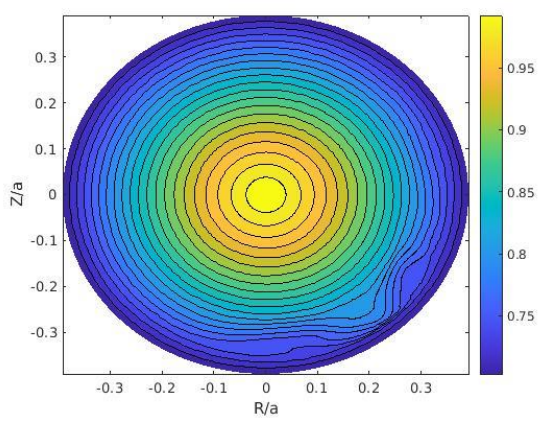

b) $t=1.21 \times 10^{-5} \tau_{R}$

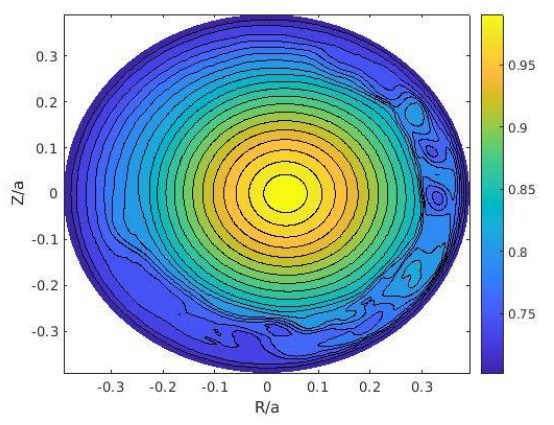




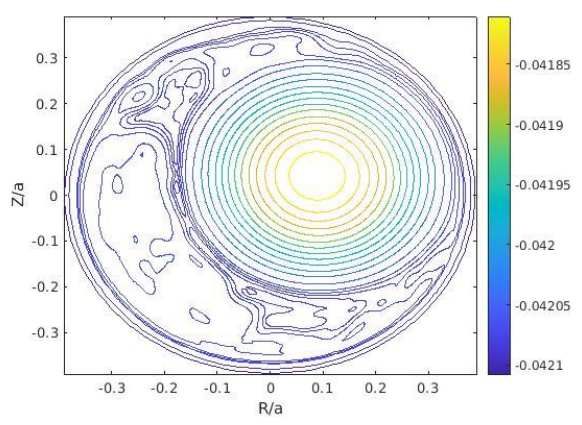

c) $t=1.37 \times 10^{-5} \tau_{R}$
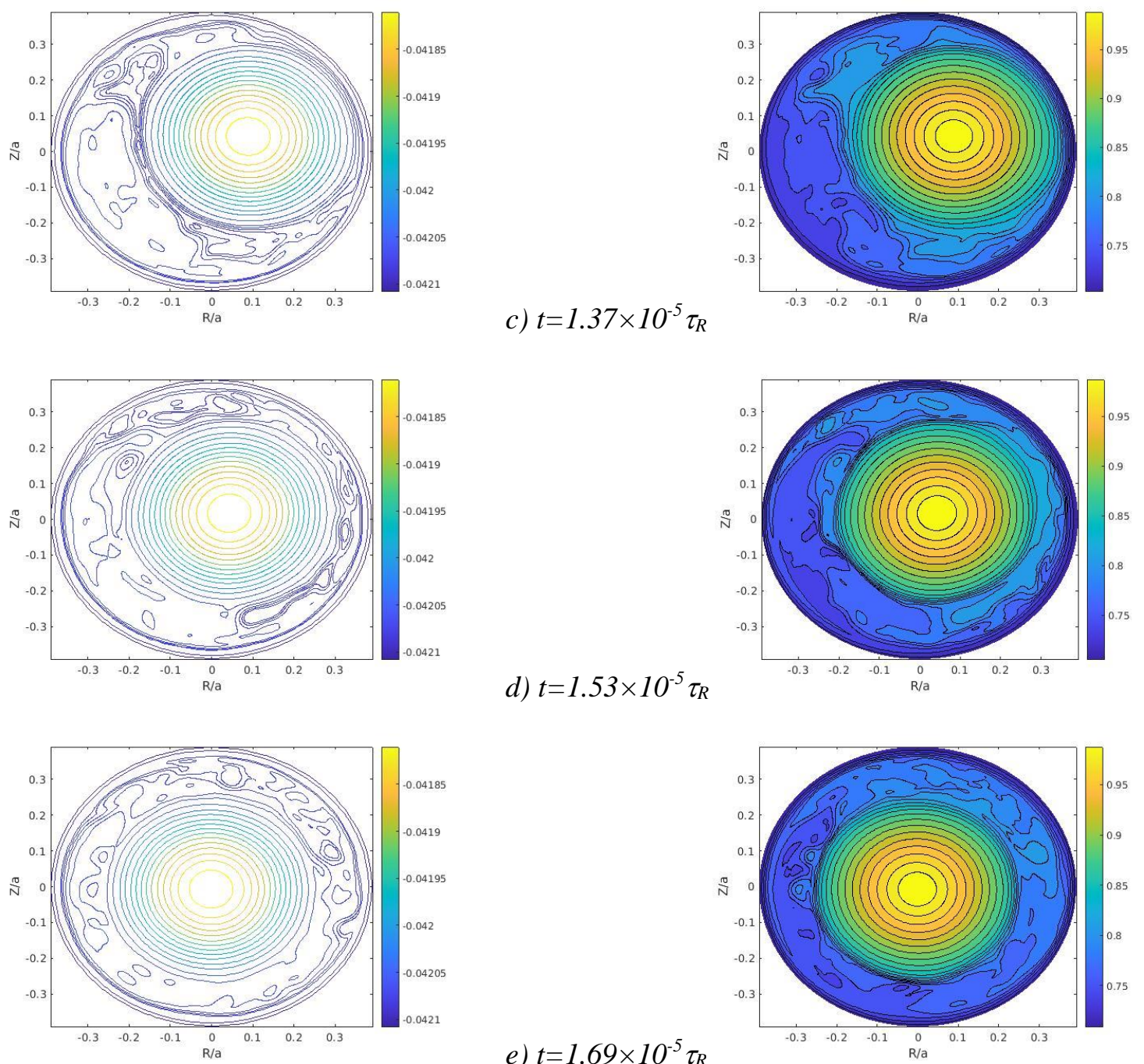

d) $t=1.53 \times 10^{-5} \tau_{R}$

e) $t=1.69 \times 10^{-5} \tau_{R}$

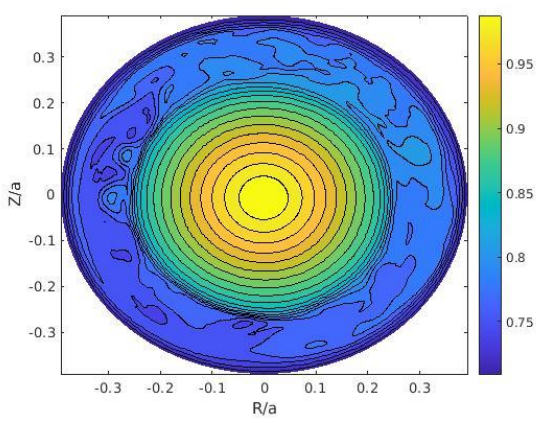

FIG. 8. Corresponding to figure 5, contours of constant helical flux (left) and electron temperature (right) at $t=1.05 \times 10^{-5}(\mathrm{a}), 1.21 \times 10^{-5}(\mathrm{~b}), 1.37 \times 10^{-5}(\mathrm{c}), 1.53 \times 10^{-5}(\mathrm{~d})$, and $1.69 \times 10^{-5} \tau_{R}(e)$ at one toroidal angle $\left(\tau_{R}=47 \mathrm{~s}\right)$. The equilibrium magnetic axis is at $R=Z=0$.

Radial profiles of $\mathrm{T}_{\mathrm{e}}(\mathrm{m} / \mathrm{n}=0 / 0)$ are shown in figure 9 at different times, $\mathrm{t}=0,1.21 \times 10^{-5}$, $1.37 \times 10^{-5}$, and $2.09 \times 10^{-5} \tau_{R}$. As already discussed above, the DTMs first cause a change of $T_{e}$ around the $\mathrm{q}=1$ surfaces marked by the two vertical dotted lines at $\mathrm{t}=1.21 \times 10^{-5} \tau_{\mathrm{R}}$ (red curve). When the IKM has grown to a large amplitude, $\mathrm{T}_{\mathrm{e}}$ drops in the central region $\left(\mathrm{t}=1.37 \times 10^{-5} \tau_{\mathrm{R}}\right.$, blue). On a longer time scale $\left(t=2.09 \times 10^{-5} \tau_{R}\right.$, green $)$, the central electron temperature recovers, while a local flattening around the $\mathrm{q}=1$ surfaces results from the DTMs. The observed moderate decrease in the central electron temperatures seen in figure 9 will be referred to as a partial crash in the following. 


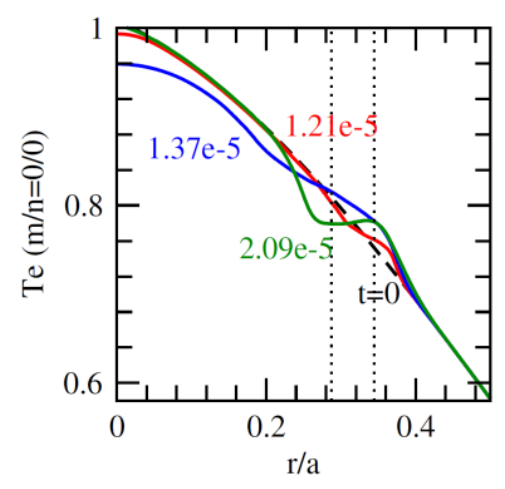

FIG. 9. Radial profiles of $T_{e}(\mathrm{~m} / \mathrm{n}=0 / 0)$ at $t=1.21 \times 10^{-5}, 1.37 \times 10^{-5}$, and $2.09 \times 10^{-5} \tau_{R}$. The dashed curve shows the radial profile of the equilibrium $T_{e}$ at $t=0$. The two vertical dotted lines show the location of the equilibrium $q=1$ surfaces.

In figure 10, the time evolution of the local radial profiles of the averaged safety factor, $\mathrm{q}_{0 / 0}$, calculated by using only the $\mathrm{m} / \mathrm{n}=0 / 0$ component poloidal field, is shown at $\mathrm{t}=0,1.21 \times 10^{-5}, 1.37 \times 10^{-5}$, and $2.09 \times 10^{-5} \tau_{\mathrm{R}}$. The q-profile is first modified around the $\mathrm{q}=1$ surfaces (red curve), indicating the change of the equilibrium plasma current density by DTMs. During the growth of the IKM, the q value decreases in the central region, accompanying the outwards motion of the plasma core (blue). Finally, only a flattening of the q-profile between the two $q=1$ surfaces remains, caused by the DTMs (green). Such a flattening of the q-profile between two resonant surfaces is very similar to observations in tokamak experiments and in the corresponding nonlinear simulations for $m / n=2 / 1$ DTMs [8].

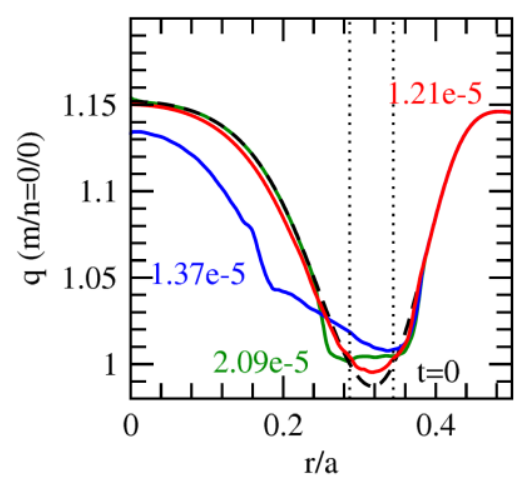

FIG. 10. Local radial profiles of $q_{0 / 0}$, the averaged safety factor calculated by using only the $m / n=0 / 0$ component poloidal field, at $t=1.21 \times 10^{-5}, 1.37 \times 10^{-5}$, and $2.09 \times 10^{-5} \tau_{R}$. The dashed curve shows the radial equilibrium $q$ profile at $t=0$. The two vertical dotted lines show the location of the original equilibrium $q=1$ surfaces.

With a smaller change of the q-profile by ECCD such that the distance between the two $q=1$ surfaces is smaller, an even smaller change in the time evolution of $\mathrm{T}_{\mathrm{e}}$ is found in calculations.

\subsection{Nonlinear results with a large distance between the resonant surfaces}

The following simulations started with the equilibrium q-profile shown by the blue curve in 
figure 2, which has a larger distance between two $\mathrm{q}=1$ surfaces.

The time evolution of the electron temperature $\left(\mathrm{T}_{\mathrm{e}}(\mathrm{m} / \mathrm{n}=0 / 0)\right)$ at the radial locations $\mathrm{r} / \mathrm{a}=0.1,0.2$, 0.3 and 0.4 is shown in figure 11 . The two $\mathrm{q}=1$ surfaces of the equilibrium q-profile are located at $\mathrm{r} / \mathrm{a}=0.234$ and 0.384 . There is again no significant change in the electron temperature at the beginning of the simulation. The electron temperature first changes around the $\mathrm{q}=1$ surfaces (green and blue curves), again due to the fast growth of DTMs. The IKM only grows up at a later time, causing a quick drop in the central electron temperature around $t=1.6 \times 10^{-5} \tau_{R}$ and a corresponding increase outside the equilibrium $\mathrm{q}=1$ surfaces $(\mathrm{r} / \mathrm{a}=0.4)$. The crash time of the central electron temperature is about $6 \times 10^{-}$ ${ }^{7} \tau_{\mathrm{R}}=28 \mu \mathrm{s}$, being comparable to the $\mathrm{W} 7-\mathrm{X}$ experimental results shown in figure 1 . This time scale is also similar to the very short sawtooth crash times observed in tokamak experiments and corresponding earlier numerical simulation results $[6,7]$.

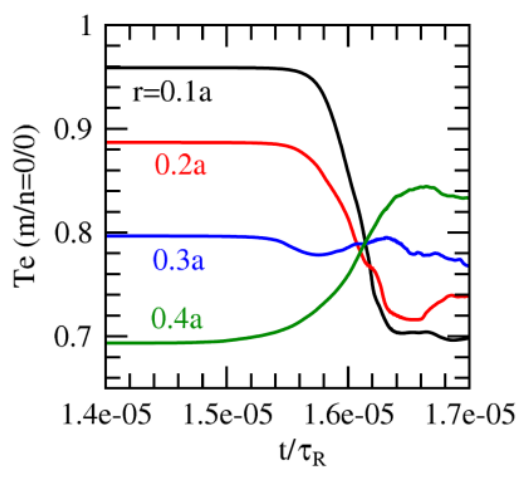

FIG.11. (left) Time evolution of the $m / n=0 / 0$ component electron temperature at the radial location $r / a=0.1$, 0.2, 0.3 and 0.4 for the equilibrium q-profile shown by the blue curve in figure 2.

Corresponding to figure 11, contours of constant helical flux (left) and electron temperature (right) are shown in figure 12 at $\mathrm{t}=1.52 \times 10^{-5}$ (a), $1.56 \times 10^{-5}$ (b), $1.59 \times 10^{-5}$ (c), $1.61 \times 10^{-5}(\mathrm{~d}), 1.63 \times 10^{-}$ ${ }^{5}(\mathrm{e}), 1.68 \times 10^{-5}$ (f), and $2.52 \times 10^{-5} \tau_{\mathrm{R}}(\mathrm{g})$ at one toroidal angle (from top to bottom). It is seen again that in addition to the early growth of DTMs localized around $q=1$ surfaces, the original core moves outwards later due to the growth of an IKM, which also affects the DTM structure. The IKM is however much stronger in this case. In a short time scale ( $28 \mu \mathrm{s})$ the original core moves to the region of the original $\mathrm{q}=1$ surfaces together with the growth of a large $m=1$ island, causing the fast crash of central electron temperature. Plasmoids are also formed during the IKM growth. The hot core disappears in a much longer time scale than the crash time. 

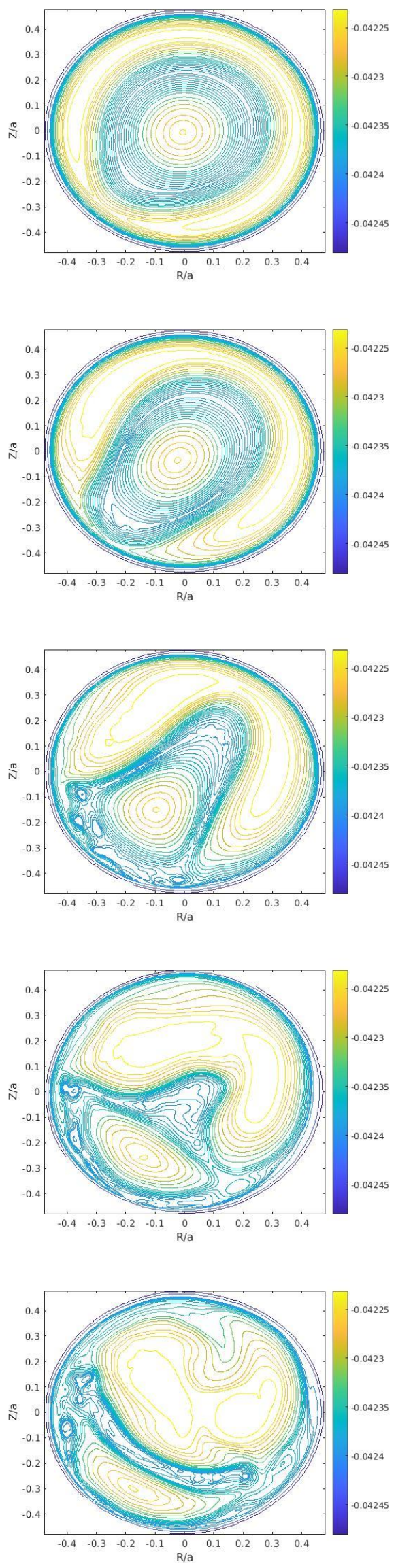

a) $t=1.52 \times 10^{-5} \tau_{R}$

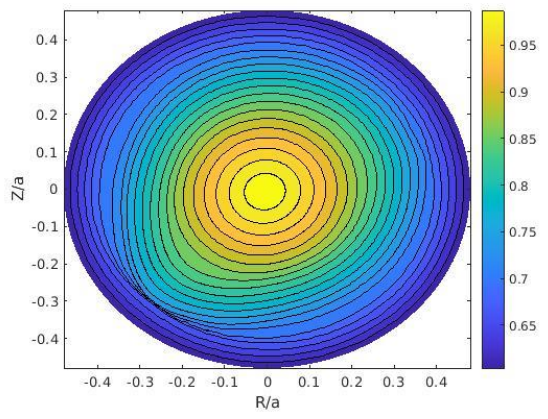

b) $t=1.56 \times 10^{-5} \tau_{R}$
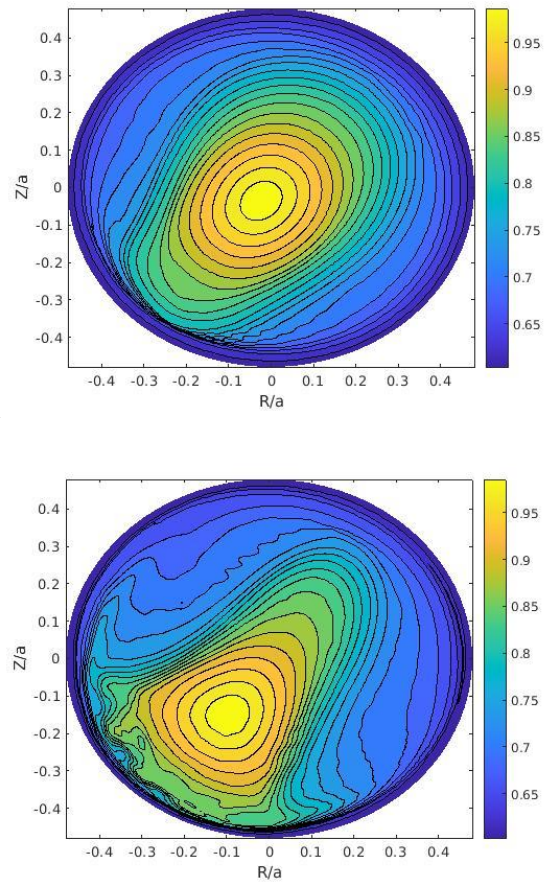

c) $t=1.59 \times 10^{-5} \tau_{R}$

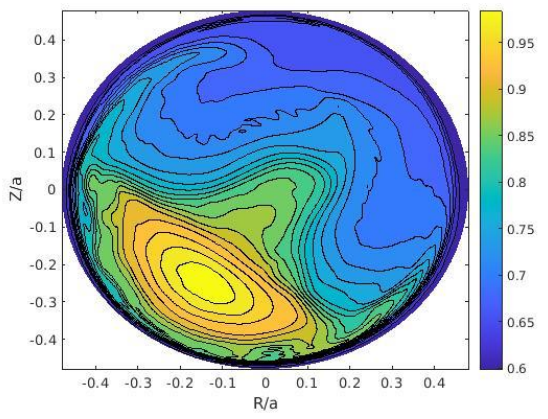

d) $t=1.61 \times 10^{-5} \tau_{R}$

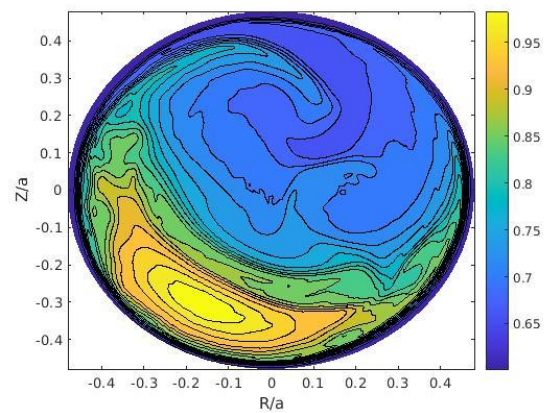




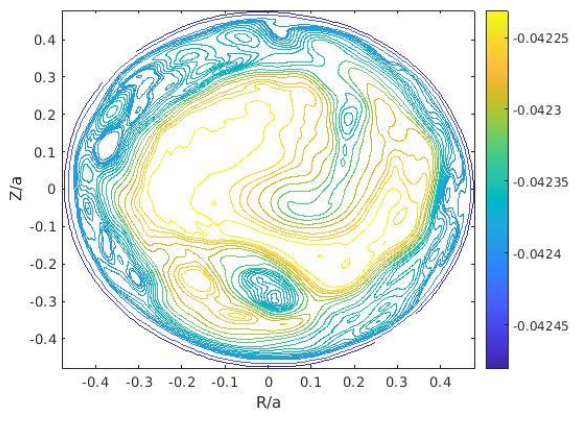

$$
\text { f) } t=1.68 \times 10^{-5} \tau_{R}
$$
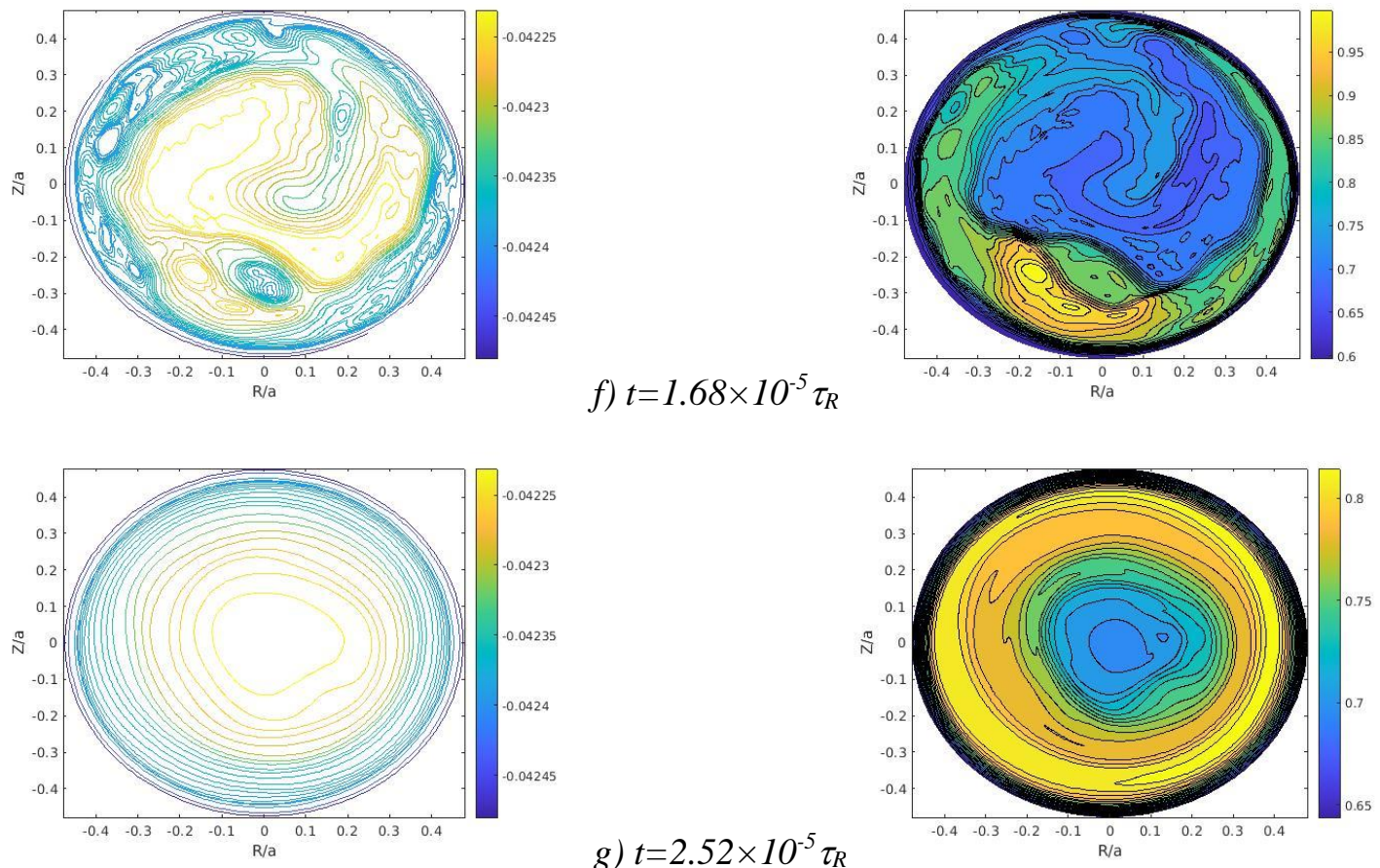

$$
\text { g) } t=2.52 \times 10^{-5} \tau_{R}
$$

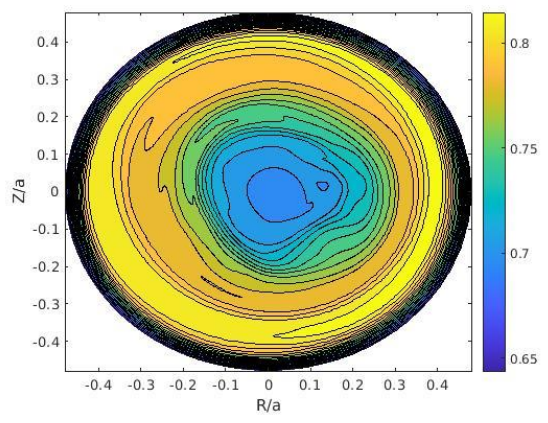

FIG. 12. Contours of constant helical flux (left) and electron temperature (right) at $t=1.52 \times 10^{-5}(\mathrm{a}), 1.56 \times 10^{-5}$ (b) $1.59 \times 10^{-5}(\mathrm{c}), 1.61 \times 10^{-5}(\mathrm{~d}), 1.63 \times 10^{-5}(\mathrm{e}), 1.68 \times 10^{-5}(\mathrm{f})$, and $2.52 \times 10^{-5} \tau_{R}(\mathrm{~g})$ at one toroidal angle. The equilibrium magnetic axis is at $R=Z=0$.

Figure 13 shows the corresponding radial profiles of the $m / n=0 / 0$ component electron temperature at different times. The DTMs first cause the change of $\mathrm{T}_{\mathrm{e}}$ around $\mathrm{q}=1$ surfaces marked by two vertical dotted lines at $\mathrm{t}=1.56 \times 10^{-5} \tau_{R}$ (red curve), and there is also a slight decrease of central $\mathrm{T}_{\mathrm{e}}$ similar to that during a partial crash. As the IKM further grows, $\mathrm{T}_{\mathrm{e}}$ drops in the central region, corresponding to the time of the outward motion of the plasma core $\left(\mathrm{t}=1.58 \times 10^{-5} \tau_{R}\right.$, blue). The time interval between the red and green curves is only $6 \times 10^{-7} \tau_{R}=28 \mu \mathrm{s}$. In this short time period, the electron temperature profile becomes completely flattened over the region from the plasma center to the $q=1$ surfaces. Such a strong temperature flattening will be referred to as a full crash in the discussion below. The electron temperature profile becomes even hollow at later times, $\mathrm{t}=1.62 \times 10^{-5} \tau_{\mathrm{R}}$ and $1.66 \times 10^{-5} \tau_{\mathrm{R}}$, due to the radial displacement of the hot core, being consistent with figure 12 .

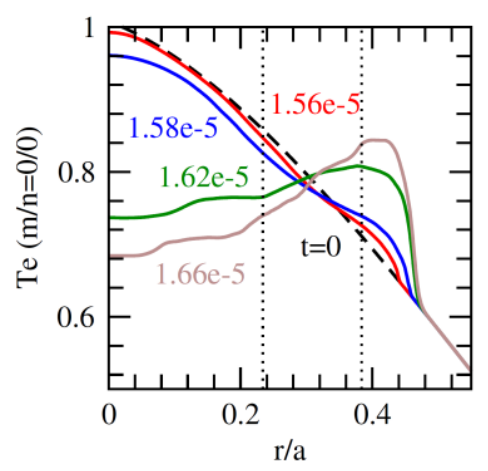


FIG. 13. Corresponding to figure 11, local radial profiles of the $m / n=0 / 0$ component electron temperature at $t=1.56 \times 10^{-5}, 1.58 \times 10^{-5}, 1.62 \times 10^{-5}$, and $1.66 \times 10^{-5} \tau_{R}$. The dashed curve shows the radial profile of the equilibrium $T_{e}$ at $t=0$. The two vertical dotted lines show the location of the equilibrium $q=1$ surfaces. The time interval between the red and green curve is $6 \times 10^{-7} \tau_{R}=28 \mu$.

The time evolution of the corresponding radial profiles of $q_{0 / 0}$ are shown in figure 14 . The flattening of the q-profile in the central region is similar to that observed in the simulations for tokamak sawtooth crashes [6,7].

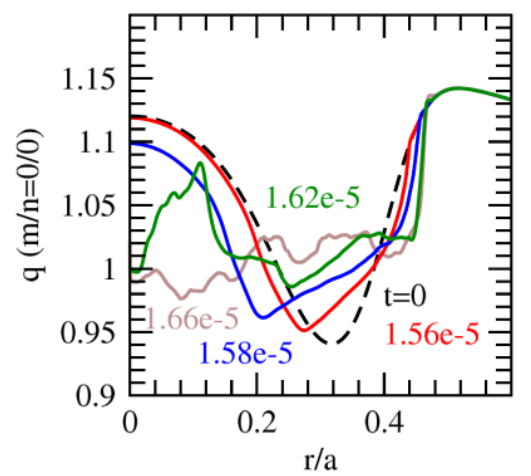

FIG. 14. Corresponding to figure 11 , radial profiles of $q_{0 / 0}$ at $t=1.56 \times 10^{-5}, 1.58 \times 10^{-5}, 1.62 \times 10^{-5}$, and $1.66 \times 10^{-}$ ${ }^{5} \tau_{R}$. The dashed curve shows the equilibrium q-profile at $t=0$.

\subsection{Regimes for partial and full crashes}

Extensive calculations have been carried out using different q-profiles defined by equation (12). The results are shown in the $\left\{\alpha_{1}, \alpha_{0}\right\}$ plane in figure 15. The red circles mark the cases for which full crashes of the electron temperature are found, similar to that shown in figure 13. The black squares are the cases for that partial crashes are found, similar to that shown in figure 9 . It is seen that for a larger value of $\alpha_{0}$ or $\alpha_{1}$, corresponding to a larger change of the original equilibrium q-profile by ECCD, full crashes of the electron temperature from the magnetic axis up to the $q=1$ surfaces are observed.

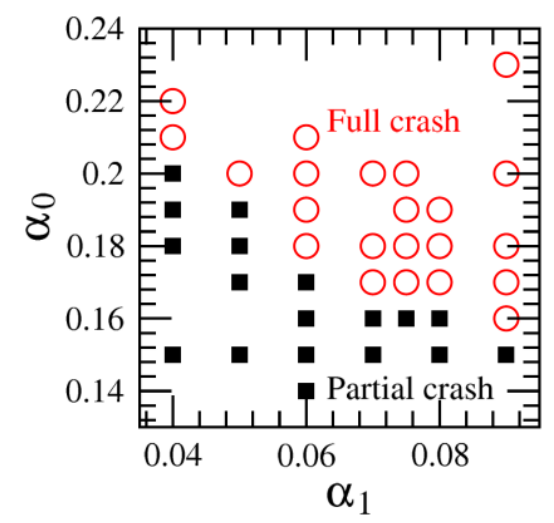

FIG. 15. Regimes for full (red circles) and partial crashes (black squares) in the $\left\{\alpha_{1}, \alpha_{0}\right\}$ plane. 


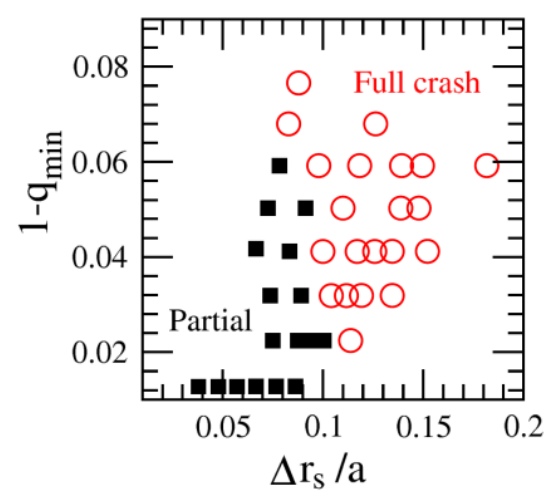

FIG. 16. The results shown in figure 15 are plotted in the $\left\{\Delta r_{s} / a,\left(1-q_{\min }\right)\right\}$ plane. The red circles (black squares) correspond to full (partial) crashes. A larger value of $\Delta r_{s}$ or (1-qmin) leads to a full crash.

The results of figure 15 are plotted in figure 16 in terms of quantities more directly related to the q-profile: the normalized distance between two $\mathrm{q}=1$ surfaces, $\Delta \mathrm{r}_{\mathrm{s}} / \mathrm{a}$, and the minimum $\mathrm{q}$-value, $\mathrm{q}_{\min }$. The red circles (black squares) again correspond to full (partial) crashes of the electron temperature. It is seen that a larger value of $\Delta \mathrm{r}_{\mathrm{s}}$ or $\left(1-\mathrm{q}_{\mathrm{min}}\right)$ results in full crashes. When approaching the boundary between full and partial crashes, the amplitude of partial crashes becomes larger, and the crash time decreases. In the opposite limit, the amplitude of partial crashes becomes reduced. The crash time of full crashes near the boundary is longer than that shown in figure 11 , reaching about $50 \mu$ s for a large value of $\Delta \mathrm{r}_{\mathrm{s}}$. The relative amplitude between the $1 / 1$ and the $\mathrm{m}>1$ components during crashes is smaller for partial crashes than that for full crashes, decreasing for a smaller value of $\Delta r_{s}$ or $\left(1-q_{\min }\right)$.

In the nonlinear phase, the $\mathrm{m} / \mathrm{n}=0 / 0$ component of the sheared poloidal plasma $(\mathbf{E} \times \mathbf{B})$ rotation is always found to be driven, although the total electromagnetic torque is zero. The driven velocity is higher for a larger value of $\Delta r_{s}$ or $\left(1-q_{\text {min }}\right)$. An example of the radial profile of the $m / n=0 / 0$ component of the poloidal plasma rotation velocity is shown in Fig. 17 at a time when the decrease of the central electron temperature is in its half way during a full crash, with $\alpha_{0}=0.19$ and $\alpha_{1}=0.06$. The original equilibrium plasma rotation velocity is zero. The amplitude of sheared plasma rotation velocity reaches $10^{5} \mathrm{a} / \tau_{\mathrm{R}}\left(\sim 10^{3} \mathrm{~m} / \mathrm{s}\right)$ at this time. This is similar to that found in the simulation of tokamak sawtooth crashes [6]. It should be noted however that for W7-X type stellarators, the plasma rotation is subjected to strong neoclassical damping, so that the crash is expected to drive the plasma rotation only temporally. 


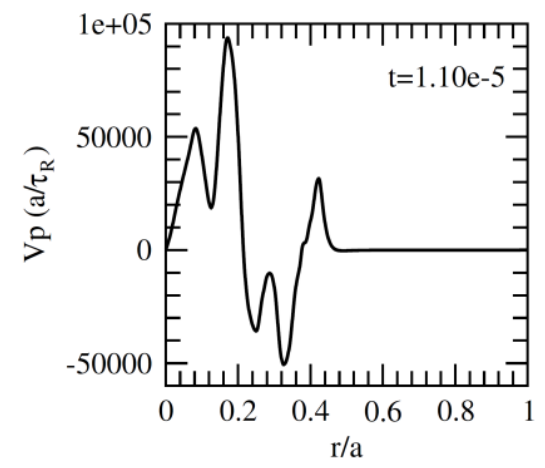

FIG. 17. Radial profile of the $\mathrm{m} / \mathrm{n}=0 / 0$ component of the poloidal plasma $(\boldsymbol{E} \times \boldsymbol{B})$ velocity at a time when the decrease of the central electron temperature is in its half way during a full crash, with $\alpha_{0}=0.19$ and $\alpha_{1}=0.06$.

\section{Discussion and summary}

In order to understand the sawtooth-like crashes of the electron temperature observed in W7-X experiments, in which ECCD leads to non-monotonic q-profiles with two q=1 surfaces inside the plasma [2,3], numerical calculations based on two-fluid equations have been carried out. For our simulations a simplified cylindrical geometry has been used. Comparisons with linear simulations in realistic W7-X geometry however showed agreement. Our nonlinear results show that both IKMs and DTMs are unstable for ECCD induced q-profiles. In the earlier phase of the simulations, high-m DTMs have larger amplitude than low-m modes, as expected from the linear results. In the later nonlinear phase, the low-m modes dominate. The growth of the IKM is dominated by the $m / n=1 / 1$ component, causing a drop of the central electron temperature. DTMs however, containing many Fourier components, flatten the local electron temperature and q-profile in the region between two q=1 surfaces. The 1/1 component also contributes to the DTMs, in addition to causing the IKM. The IKM and DTMs affect each other due to nonlinear mode coupling, which results in the coupling of all components of different mode numbers.

Considering of the possible difference between the q-profiles utilized in our simulations and experimental ones, a class of parametrized q-profiles with two $q=1$ surfaces inside the plasma are investigated. Two types of crashes, partial and full crashes, are found from our numerical results, depending on the distance between the two equilibrium $q=1$ surfaces and the minimum $q$ value. As is known, the free magnetic energy driving DTMs comes mainly from the region between the two resonant surfaces [9]. A smaller distance between two $q=1$ surfaces or a smaller value of (1- $\left.\mathrm{q}_{\min }\right)$ thus corresponds to less free magnetic energy available, which can be quickly released by the fast growing DTMs. As a result, the value of $\mathrm{q}_{\min }$ is increased to about one as shown in figure 10, and the IKM can only temporally grow to a moderate amplitude and cause a partial crash. When the distance of two $\mathrm{q}=1$ surfaces or the value of $\left(1-\mathrm{q}_{\mathrm{min}}\right)$ is sufficiently large, the value of $\mathrm{q}_{\min }$ remains to be below 1 during 
the DTMs growth, as shown in figure 14, and the free energy is large enough to drive the IKM to a large amplitude, causing a full crash.

Each of our calculation starts from a given equilibrium q-profile. In real experiments ECCD acts as an effective parallel electric field to gradually increase the value of $\left(1-\mathrm{q}_{\mathrm{min}}\right)$ or the distance of two $\mathrm{q}=1$ surfaces [2,3]. One would therefore expect that partial crashes will happen before a full crash. It is important to note that two kinds of crashes, with large and small amplitudes, were indeed seen in W7-X experiments [10]. Precursors were also found before the crashes [10]. Detailed data analysis to characterize the large and small crashes will be reported separately in an experimental paper. Due to the formation of $q=1$ surface in W7-AS experiments with ECCD, the sawtooth-like oscillations preceded by $m / n=1 / 1$ mode activity had also been observed [11 and reference therein].

When neglecting the parallel ion flow, about the same numerical results have been found, showing that it is not important for the mode growth. When taking a smaller (by 1/10 times) perpendicular transport coefficients or a larger ratio (by 10 times) between the parallel and perpendicular electron heat conductivity, similar results are also found.

It should be emphasized again that some high-n components have not been included in our calculations due to the simplified geometry. To study the high-n modes and their coupling associated with stellarator geometry, a three-dimensional code is required [5]. As low-n components dominate the crash of the central electron temperature, our results approximately agree with experimental observations [2,3], revealing a simplified way to look into nonlinear low-n modes in stellarator plasmas.

Although there is no equilibrium plasma current in our model except for that due to ECCD, the simulated crashes are similar to the tokamak case, indicating that the q-profile is the most important factor in determining the sawtooth instability. As the q-profile is affected by the deposition location and the amount of power of the electron cyclotron waves, ECCD can be utilized as a tool to generate moderate sawtooth crashes for reducing impurity accumulation in the center of stellarator plasmas if needed.

In summary, numerical calculations based on nonlinear two-fluid equations have been carried out to investigate the sawtooth-like crashes of the electron temperature observed in W7-X experiments, which have non-monotonic 1 -profiles with two $t=1$ surfaces in the plasma core region due to ECCD. Using experimental parameters as input, fast crashes of the electron temperature in the central region are found in our numerical calculations. They are caused by the growth of the internal kink mode coupled to double tearing modes. Two type of crashes, full and partial crashes, are found, depending on the distance between the two equilibrium $\mathrm{q}=1$ surfaces and the minimum $\mathrm{q}$ value. 


\section{Acknowledgements}

This work has been carried out within the framework of the EUROfusion Consortium and has received funding from the Euratom research and training programme 2014-2018 and 2019-2020 under

grant agreement number 633053. The views and opinions expressed herein do not necessarily reflect those of the European Commission. 


\section{References}

[1] Geiger J. et al 2013 Plasma Phys. Control. Fusion 55, 014006.

[2] Wolf R.C. et al 2017 Nucl. Fusion 57, 102020.

[3] Wolf R.C. et al 2019 Phys. Plasmas 26, 082504.

[4] Von Goeler S. et al 1974 Phys. Rev. Lett. 331201.

[5] Strumberger E. and Günter S. 2017 Nucl. Fusion 57, 016032.

[6] Yu Q., Günter S. and Lackner K. 2015 Nucl. Fusion 55113008.

[7] Günter S., Yu Q., Lackner K., Bhattacharjee A. and Huang Y-M. 2015 Plasma Phys. Control. Fusion 57014017.

[8] Günter S. et al 2000 Nucl. Fusion 401541.

[9] Wesson, J.A. 1978 Nucl. Fusion 1887.

[10] Zanini M. et al , 2019 EPJ Web of Conferences 203, 02013 https://www.epj-conferences.org/articles/epjconf/pdf/2019/08/epjconf_ec2018_02013.pdf [11] Hirsch M. et al Plasma Phys. Control. Fusion 50 (2008) 053001. 